Conclusion This meta-analysis has found evidence for a protective effect of later puberty for testicular cancer risk.

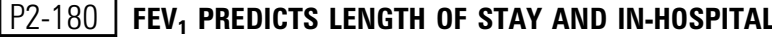 MORTALITY IN PATIENTS UNDERGOING CARDIAC SURGERY}

doi:10.1136/jech.2011.142976j.15

${ }^{1} \mathrm{D}$ McAllister, ${ }^{2} \mathrm{~S}$ Wild, ${ }^{3} \mathrm{~J}$ Maclay, ${ }^{4} \mathrm{~A}$ Robson, ${ }^{2} \mathrm{D}$ Newby, ${ }^{2} \mathrm{~W}$ MacNee, ${ }^{4} \mathrm{~A}$ Innes, ${ }^{4} \mathrm{~V}$ Zamvar, ${ }^{2} \mathrm{~N}$ Mills. ${ }^{1} \mathrm{NHS}$ Fife, Fife, UK; ${ }^{2}$ University of Edinburgh, Edinburgh, UK; ${ }^{3} \mathrm{NHS}$ Greater Glasgow, Glasgow, UK; ${ }^{4} \mathrm{NHS}$ Lothian, Lothian, UK

Background Older patients are increasingly being referred for cardiac surgery, consequently novel prognostic markers which reflect physiological reserve and severity of co-morbid disease are now required. Forced Expiratory Volume in one second $\left(\mathrm{FEV}_{1}\right)$ is a robust accurate measure of pulmonary physiology and predicts all-cause mortality, yet the relationship between $\mathrm{FEV}_{1}$ and outcome in patients undergoing cardiac surgery is unknown. We hypothesised that $\mathrm{FEV}_{1}$ would predict mortality and length of hospital stay following cardiac surgery.

Methods In a retrospective cohort design, records for 2241 consecutive patients undergoing coronary artery bypass grafting and/or valve surgery from 2001 to 2007 were selected from a regional cardiac surgery database and linked to a regional spirometry database. Generalised linear models of the association between $\mathrm{FEV}_{1}$ and length of hospital stay and mortality were adjusted for age, sex, height, body mass index, socioeconomic status, smoking, cardiovascular risk factors, chronic pulmonary disease, and type and urgency of surgery. $\mathrm{FEV}_{1}$ was compared to an established risk prediction model, the EuroSCORE.

Results Spirometry was performed in 2082 cardiac surgery patients (93\%) whose mean (SD) age was 67 (10) years. Median hospital stay was 3-days longer in patients in the lowest compared to the highest quintile for $\mathrm{FEV}_{1}$, 1.35-fold higher (95\% CI 1.20 to 1.52; $\mathrm{p}<0.001$ ). The adjusted OR for mortality was increased 2.11 -fold (95\% CI 1.45-3.08; $\mathrm{p}<0.001)$ per SD decrement in $\mathrm{FEV}_{1}(800 \mathrm{ml}) . \mathrm{FEV}_{1}$ improved discrimination of the EuroSCORE for mortality.

Conclusions Reduced $\mathrm{FEV}_{1}$ strongly predicts increased length of stay and in-hospital mortality following cardiac surgery.

\section{P2-181 RECONSTRUCTING THE HISTORICAL INCIDENCE OF HEPATITIS C INFECTION AMONG SCOTLAND'S IDUS}

\section{doi:10.1136/jech.2011.142976j.16}

${ }^{1,2} \mathrm{~S}$ McDonald, ${ }^{* 2,3} \mathrm{M}$ Kretzschmar, ${ }^{1,4} \mathrm{~S}$ Hutchinson, ${ }^{1} \mathrm{D}$ Goldberg. ${ }^{1}$ Health Protection Scotland, Glasgow, UK; ${ }^{2}$ Julius Centre for Health Sciences \& Primary Care, University Medical Centre, Utrecht, The Netherlands; ${ }^{3}$ Center for Infectious Disease Control, RIVM, Bilthoven, The Netherlands; ${ }^{4}$ University of Strathclyde, Glasgow, UK

Introduction The historical incidence of hepatitis C virus (HCV) infection in Scotland's injecting drug user (IDU) population is unknown. From a public health perspective, accurate data on incidence rates over time would improve our understanding of the effects of harm reduction measures initiated in the late 1980s (needle/syringe exchanges, methadone treatment) on the transmission of HCV among Scotland's IDUs. Because HCV-antibody testing only commenced in 1991, and positive diagnoses are often made many years after infection, trends in incidence cannot easily be inferred from trends in the time-series of diagnosed cases.

Methods We applied back-calculation methods to reconstruct HCV incidence using data available on the national HCV Diagnosis database (1991-2009; $n=25000)$, estimates from the literature regarding time to seroconversion and mortality rates, and the estimated distribution of time between injection debut and diagnosis, derived via record-linkage between the HCV Diagnosis and the Scottish Drugs Misuse databases.

Results Approximately 50000 IDUs were estimated to have been infected with HCV during 1960-2009. The shape of the incidence curve varied with region, but a peak in annual incidence was estimated to have occurred around 1993. The model projected approximately 1700 new HCV diagnoses per year over the coming 5 years. Sensitivity analyses were also conducted to explore assumptions regarding mortality rate and the proportion of infected IDUs that are never diagnosed.

Conclusion These incidence data are important for evaluation of the impact of harm reduction initiatives, for informing national public health planning, and for projecting the future burden of $\mathrm{HCV}$ related severe liver disease.

\section{P2-182 THE INFLUENCE OF HEPATITIS C AND ALCOHOL ON LIVER- RELATED MORBIDITY AND MORTALITY IN GLASGOW}

doi:10.1136/jech.2011.142976j.17

${ }^{1,2} \mathrm{~S}$ McDonald, ${ }^{* 1,2} \mathrm{~S}$ Hutchinson, ${ }^{3} \mathrm{P}$ Mills, ${ }^{2,4} \mathrm{~S}$ Bird, ${ }^{5} \mathrm{~S}$ Cameron, ${ }^{6} \mathrm{~J}$ Dillon, ${ }^{1} \mathrm{D}$ Goldberg. ${ }^{1}$ Health Protection Scotland, Glasgow, UK; ${ }^{2}$ Department of Mathematics \& Statistics, University of Strathclyde, Glasgow, UK; ${ }^{3}$ Gartnavel General Hospital, Glasgow, UK; ${ }^{4}$ MRC Biostatistics Unit, Institute of Public Health, Cambridge, UK; ${ }^{5}$ West of Scotland Specialist Virology Centre, Gartnavel General Hospital, Glasgow, UK; ${ }^{6}$ Ninewells Hospital \& Medical School, Dundee, UK

Introduction Infection with the hepatitis $\mathrm{C}$ virus (HCV) is associated with the development of severe liver disease, but cofactors - namely alcohol abuse - in Scotlands HCV-positive population complicate estimation of the unique contribution of HCV. We compared the risk of hospital admission/death for a liver-related cause in a large cohort of Glasgow's injecting drug users (IDUs) testing HCV-positive, with IDUs testing HCV-negative.

Methods Data for 6566 current/former IDUs who had been tested for anti-HCV and/or HCV RNA in Greater Glasgow health board between 1993 and 2007 were linked to the national hospitalisation database and deaths registry to identify all admissions and deaths from a liver-related condition. RRs were estimated using Cox regression for recurrent events.

Results The risk of hospitalisation/death from a liver-related or an alcoholic liver-related condition following HCV testing was greater for those IDUs with no prior alcohol-related hospitalisation who tested positive [adjusted hazard ratio (HR) $=3.2,95 \%$ CI 1.5 to 6.7 ; $4.9,95 \%$ CI 1.8 to 13.1 , respectively], compared with those who tested anti-HCV negative, but not for those IDUs with a prior alcohol admission ( $\mathrm{HR}=0.8,95 \%$ CI 0.4 to 1.5 ; $0.8,95 \%$ CI 0.4 to 1.6). There was little evidence for an increased risk of hospitalisation/death for an exclusively non-alcoholic liver condition for those testing positive ( $\mathrm{HR}=1.5,95 \% \mathrm{CI} 0.8$ to 2.7 ), after adjustment for previous alcohol-related admission.

Conclusion Within Glasgow's IDU population, HCV positivity is associated with an increased risk of a liver-related outcome, but this is not observed for those IDUs whose problem alcohol use already increases their risk.

\section{P2-183 PATTERNS OF ANTERIOR AND POSTERIOR CARIES BY SOCIOECONOMIC STATUS IN 3-YEAR-OLD CHILDREN}

doi:10.1136/jech.2011.142976j.18

${ }^{1}$ A D McMahon, ${ }^{*}{ }^{2} Y$ Blair, ${ }^{2} \mathrm{D}$ R McCall, ${ }^{1} \mathrm{~L}$ M D Macpherson. ${ }^{1}$ Glasgow University Dental School, Glasgow, Scotland, UK; ${ }^{2}$ NHS Greater Glasgow and Clyde, Glasgow, Scotland, UK

The aim was to examine if anterior/posterior patterns of decayed missing and filled teeth in 3-year-old children in Greater Glasgow 